\title{
Importance Socioculturelle Des Produits Forestiers Non Ligneux Du Massif Forestier D’agoua Au Benin
}

\author{
Wotto Alex \\ Laboratoire d'Ecologie Appliquée (LEA), Faculté des Sciences \\ Agronomiques, Université d’Abomey-Calavi, Cotonou, Bénin \\ Gbaguidi Aholidji Humbert Ulrich \\ Laboratoire de Biogéographie et d'Expertise Environnementale (LABEE), \\ Université d'Abomey-Calavi, Bénin \\ Vissoh Ahotondji Sylvain \\ Laboratoire d'Etudes des Dynamiques Urbaines et Régionales (LEDUR), \\ Université d'Abomey-Calavi, Bénin
}

doi: 10.19044/esj.2017.v13n14p123 URL:http://dx.doi.org/10.19044/esj.2017.v13n14p123

\begin{abstract}
The aim of this research is to analyze the socio-cultural importance of non-ligneous forest products (NWFPs) in the forest of Agoua, located in the department of Hills in Benin. This research led to an inventory of NWFPs and ethnobotanical surveys among 50 foresters, 62 NWFP resellers, 46 consumers and 12 phytotherapists. The data collected relate to the plant species used as NWFPs by populations living on the massif of Agoua, to endogenous knowledge and to the use values of NWFPs. Thus, 105 plant species distributed in 48 families have been inventoried within the forest massif of Agoua. The determination of the different categories of NWFP uses revealed that 61 (58.09\%) of the total number of plant species surveyed were used in traditional medicine, 30 in food (28.57\%), 28 for various domestic uses (26.57\%), 8 for building materials (7.61\%), 6 for packaging (5.71\%) and 4 for coloring (3.8\%). The organs taken by the populations vary from one species to another and according to the use they make of them. In fact, it is the leaves, fruits, barks, flowers and roots that are used by the populations bordering the forest of Agoua. The excessive exploitation of NWFPs by the populations bordering on the Agoua forest is the cause of the decline and disappearance of these plant species with a high genetic and socio-cultural potential. The aggravation of this phenomenon with population growth may be detrimental to the sustainability of this forest ecosystem. In order to reduce the effects of such use, it is necessary to implement methods and collection rates that respect the sustainability of these resources.
\end{abstract}


Keywords: Agoua in Benin, forest massif, non-wood forest products, use values

\section{Résumé}

La présente recherche vise à analyser l'importance socioculturelle des Produits Forestiers Non Ligneux (PFNL) du massif forestier d'Agoua, situé dans le département des Collines au Bénin. Cette recherche a conduit à la réalisation d'un inventaire des PFNL et d'enquêtes ethnobotaniques auprès de 50 exploitants forestiers, 62 revendeurs des PFNL, 46 consommateurs et 12 phytothérapeutes. Les données collectées sont relatives aux espèces végétales utilisées comme PFNL par les populations riveraines du massif d'Agoua, aux connaissances endogènes et aux valeurs d'usage des PFNL. Ainsi, 105 espèces végétales réparties en 48 familles ont été inventoriées au sein du massif forestier d'Agoua. La détermination des différentes catégories d'utilisations des PFNL a révélé que sur le nombre total d'espèces végétales recensées, 61 (soit 58,09 \%) sont utilisées dans la médecine traditionnelle, 30 dans l'alimentation (28,57 \%), 28 pour divers usages domestiques (26,57 \%), 8 comme matériaux de construction (7,61 \%), 6 comme emballage (5,71\%) et 4 comme colorant $(3,8 \%)$. Les organes prélevés par les populations varient d'une espèce à une autre et en fonction de l'usage qu'elles en font. En effet, ce sont les feuilles, les fruits, les écorces, les fleurs et les racines qui sont utilisés par les populations riveraines de la forêt d'Agoua. La trop grande exploitation des PFNL par les populations riveraines du massif forestier d'Agoua est à l'origine de l'amenuisement et de la disparition de ces espèces végétales à forte potentialité génétique et socioculturelle. L’aggravation de ce phénomène avec l'accroissement démographique peut être néfaste pour la durabilité de cet écosystème forestier. Pour réduire les effets d'une telle utilisation, il est nécessaire de mettre en œuvre des méthodes et des taux de collecte respectant la durabilité de ces ressources.

Mots clés: Agoua au Bénin, massif forestier, produits forestiers non ligneux, valeurs d'usage.

\section{Introduction}

En Afrique tropicale, le niveau de pauvreté relativement élevé ainsi que l'instabilité des productions agricoles constituent des facteurs qui incitent les populations rurales, mais aussi urbaines à l'utilisation des Produits Forestiers Non Ligneux (PFNL) comme alternative de subsistance et source de revenus. Ces ressources participent activement à leur vie quotidienne en assurant des fonctions culturelles, de régulation et de production (Yelkouni, 2004). Cependant, le couvert végétal ainsi que le 
patrimoine des espèces pourvoyeuses de PFNL se dégradent progressivement, en raison notamment de leur utilisation non rationnelle. En effet, face à l'explosion démographique et à l'accroissement de la pression exercée sur la terre, certaines espèces qui auraient pu être d'une grande utilité ont disparu ou sont devenues très rares (Riley et Brokensha, 1988).

A l'instar des autres massifs forestiers du Bénin, celui d'Agoua, situé dans la Commune de Bantè, au centre du pays, subit une forte pression démographique. Les prélèvements des espèces végétales utilisées comme PFNL par les populations riveraines de cette forêt ont atteint un seuil critique (PAMF, 2007). En effet, l'exploitation des espèces végétales de ce massif se fait de façon abusive sans aucun souci de domestication (Lokonon, 2002). Cet état de choses pourrait, à terme, mettre en péril la pérennité de ces essences qui sont pourtant importantes pour les populations locales. La commercialisation des PFNL procure des revenus substantiels leur permettant de faire face aux divers besoins (Sokpon et Lejoly, 1996). Aussi, la consommation des PFNL par les ménages contribue à la diversification de leur alimentation (Sokpon et Lejoly, 1996 ; Assogbadjo, 2000 et Fandohan et al., 2010).

La présente recherche, en complément aux études effectuées sur les PFNL au Bénin, vise à déterminer l'importance socioculturelle des Produits Forestiers Non Ligneux (PFNL) du massif forestier d'Agoua pour les populations locales.

\section{Matériels et méthodes}

\subsection{Cadrage géographique de l'étude}

La forêt classée d'Agoua est située dans la commune de Bantè dans le département des Collines (Bénin), entre $8^{\circ} 25^{\prime}$ et $8^{\circ} 50^{\prime}$ de latitude nord et entre $1^{\circ} 40^{\prime}$ et $2^{\circ} 25^{\prime}$ de longitude est (Figure 1 ). Elle couvre une superficie d'environ (75300 ha). 


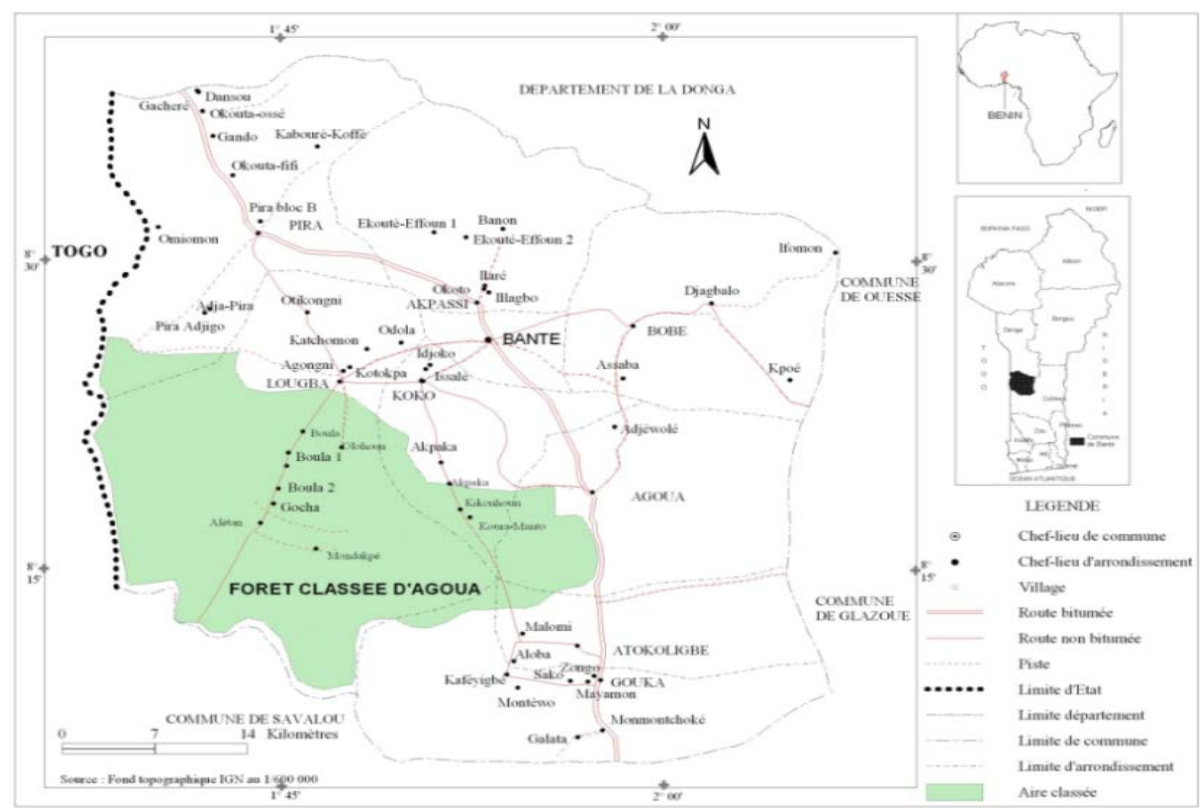

Figure 1 : Situation géographique de la forêt classée d’Agoua

Ce massif forestier bénéficie d'un climat de type soudanien humide marqué par deux saisons. Une saison sèche de novembre à mars et une saison pluvieuse d'avril à octobre. La pluviométrie annuelle est de $1100 \mathrm{~mm}$ répartie, en moyenne sur 97 jours. Les moyennes mensuelles thermiques sont comprises entre $25^{\circ} \mathrm{C}$ et $30^{\circ} \mathrm{C}$ et les écarts thermiques journaliers sont faibles (Adam et Boko, 1993). L'humidité relative est de $31 \%$ au maximum. L'insolation totale pour l'année atteint 2305 heures avec un maximum en mars (237 heures) et un minimum en août (88 heures) ASECNA (2016). Le contexte climatique du massif forestier d'Agoua est donc favorable au développement de la végétation qui ne souffre d'aucune limitation pour son alimentation hydrique et minérale. En effet, dans ce massif se trouve une diversité de formations végétales riches en PFNL qui contribuent significativement à l'amélioration des conditions de vie des populations riveraines (PAMF, 2007).

Les écosystèmes forestiers occupent 21269 hectares de la superficie totale du massif forestier d'Agoua. En effet, la superficie de la forêt dense est de 1025 hectares, celles de la forêt galerie, de la forêt claire et de la savane boisée sont respectivement de 15567 hectares et 4677 hectares (figure 2). 


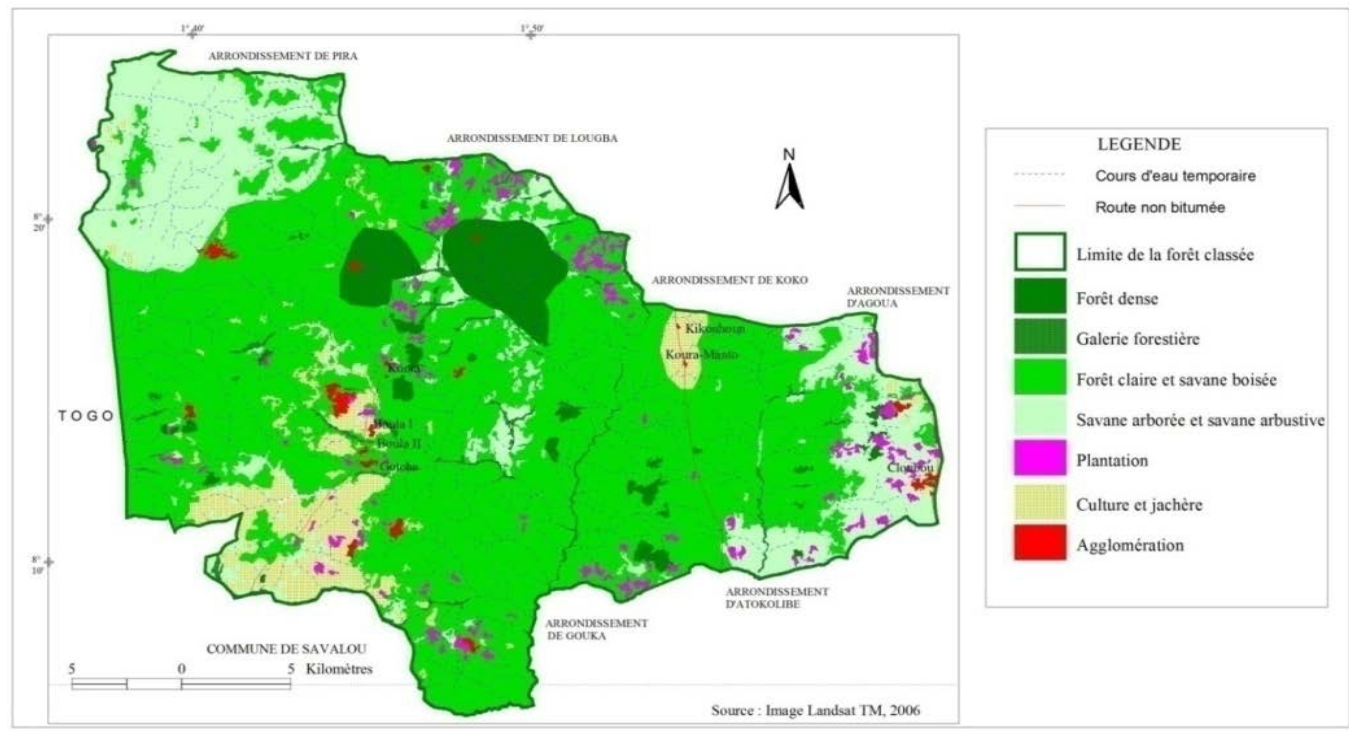

Figure 2 : Eléments d’occupation du sol de la forêt classée d’Agoua (2015)

Les espèces les plus importantes appartenant à ces formations naturelles sont: le faux iroko (Antiaris toxicaria), le fromager (Ceiba pentandra), le néré (Parkia biglobosa), le baobab (Adansonia digitata), le rônier (Borasus aethiopum), l'iroko (Milicia excelsa), le Samba (Triplochiton scleroxylon), le faux karité (Lophira lanceslata), le Rikio des montagnes (Uapaca togoensis), le Cèdre des zones sèches (Pseudocedrela kotschyi). Le reste de la superficie, soit les 66,34 \%, est couvert en grande partie de mosaïques de cultures et de jachères (2778 hectares), des plantations (10716 hectares) de teck (Tectona grandis) et d'anacardiers (Anacardium occidentalis) appartenant soit à l'Etat, soit à des privés (Colyn et al., 2007) et des agglomérations (76 hectares). Les plus grandes plantations sont celles d'anacardiers installées par la Société Nationale pour le Développement Forestier (SNAFOR) depuis 1965. La population habitant les localités riveraines du massif forestier d'Agoua est celle de la Commune de Bantè. Elle est inégalement répartie sur l'ensemble du territoire. Une projection de cette population effectuée sur la base des données du Recensement Général de la Population et de l'Habitation (RGPH) de 2013 a montré qu'elle devrait passer de 107181 habitants en 2013 à 121931 habitants en 2017.

On note plusieurs groupes socioculturels que sont par ordre d'installation dans la localité, les Isha, les Ifè, les Idasha, les Mahi, les Fon, les Djerma, les Holli, les Peulh, les Somba, les Lokpa, les Kotokoli, les Adja.

Ces différents groupes socioculturels s'adonnent à de nombreuses activités, dont l'agriculture qui constitue la principale source de revenus pour 
la majeure partie de la population. D’après le CeCPA Bantè (2015), l'agriculture est faite de cultures vivrières (maïs, riz, niébé), de tubercules (igname, manioc) et de cultures de rente : coton et anacarde (CeCPA Bantè, 2015). Outre la production végétale, les activités menées dans le secteur primaire sont entre autres : l'élevage, la pêche, l'exploitation forestière. Par ailleurs, les échanges commerciaux sont facilités par un réseau routier local plus ou moins praticable et la Route Nationale Inter Etats ${ }^{\circ} 1$ (CotonouOuagadougou) qui traverse la commune.

\subsection{Matériel de collecte des données}

Pour les travaux de terrain, le matériel d'inventaire ci-après a été utilisé :

\subsubsection{Matériel biologique}

Le matériel biologique de base de cette recherche est constitué par les Produits Forestiers Non Ligneux recensés dans le massif forestier d'Agoua.

\subsubsection{Matériel de terrain}

Le matériel de terrain pour la collecte des données se compose de:

- fiches d'enquête à l'endroit des populations riveraines ;

- penta décamètre pour l'installation des placeaux ;

- papiers journaux et des chemises dossiers pour la confection d'herbiers pour les espèces non identifiées sur le terrain ;

- lexique flore du Bénin de de Souza et la flore analytique du Bénin ;

- récepteur GPS (GARMIN OREGON 450) pour géo référencier les placeaux ;

- calculatrice scientifique.

\subsection{Méthodes de collecte des données}

Dans le cadre de cette recherche, la taille des relevés a été déterminée en tenant compte des travaux effectués en milieu tropical par plusieurs auteurs (Sinsin, 1993 ; Oumorou, 2003 ; Djego, 2006 ; Toko, 2008 ; Arouna, 2012) qui ont utilisé des surfaces variant entre 100 et $1000 \mathrm{~m}^{2}$ selon les formations végétales et les strates. Selon ces auteurs, dans les strates arbustive et arborée, prises en compte par cette recherche, doivent être installés des placeaux carrées de $900 \mathrm{~m}^{2}$; 250 placeaux carrés de 30 m x 30 $\mathrm{m}$ ont donc été installés dans les différentes formations végétales du massif forestier d'Agoua. A l'intérieur de chaque placeau, il a été effectué l'inventaire systématique des Produits Forestiers Non Ligneux recherchés par les populations, selon la méthode d'analyse phytosociologique basée sur le principe d'homogénéité floristique de la surface étudiée. Les relevés ont été effectués suivant la méthode sigmatiste de Braun-Blanquet (1932), à 
l'aide des fiches de relevé élaborées pour la circonstance. Les données collectées concernent essentiellement les produits Forestiers Non Ligneux.

Outre la collecte des données d'inventaire, des enquêtes socioculturelles ont été menées systématiquement dans les neuf villages riverains de la forêt. Au total, 170 personnes âgées de 15 ans et plus ont été enquêtées au moyen d'un questionnaire. La limitation de l'âge à 15 ans tient compte du fait que, selon les populations riveraines, pour avoir une bonne connaissance des PFNL, il faut disposer d'une certaine maturité physique et culturelle et c'est l'âge minimum pour acquérir ces atouts au niveau de ces villages. Tous les deux sexes ont été interrogés. L’aspect sexe a été intégré en vue de comparer les connaissances des PFNL entre sexes.

Les techniques utilisées pour collecter les données sont les entretiens individuels et de groupe (Tableau 1).

Tableau 1 : Effectifs des personnes enquêtées par village

\begin{tabular}{|c|c|c|c|c|c|c|c|c|c|c|}
\hline \multirow{3}{*}{$\begin{array}{c}\text { Villages } \\
\text { riverains } \\
\text { enquêtés }\end{array}$} & \multirow{2}{*}{\multicolumn{4}{|c|}{ Axe Otola-Agoua }} & \multirow{2}{*}{\multicolumn{5}{|c|}{ Axe Akatakou-Faïmbè }} & \multirow{3}{*}{$\begin{array}{c}\text { Tot } \\
\text { al }\end{array}$} \\
\hline & & & & & & & & & & \\
\hline & $\begin{array}{l}\text { Oto } \\
\text { la }\end{array}$ & $\begin{array}{l}\text { Malom } \\
\text { i }\end{array}$ & $\begin{array}{l}\text { Atokoli } \\
\text { bè }\end{array}$ & $\begin{array}{l}\text { Agou } \\
\text { a }\end{array}$ & $\begin{array}{l}\text { Akatak } \\
\text { ou }\end{array}$ & $\begin{array}{l}\text { Ko } \\
\text { ko }\end{array}$ & $\begin{array}{l}\text { Tob } \\
\text { è }\end{array}$ & $\begin{array}{l}\text { Loug } \\
\text { ba }\end{array}$ & $\begin{array}{l}\text { Faïmb } \\
\text { e }\end{array}$ & \\
\hline $\begin{array}{l}\text { Exploitants } \\
\text { forestiers }\end{array}$ & 4 & 5 & 8 & 9 & 6 & 5 & 8 & 3 & 2 & 50 \\
\hline Commerçants & 3 & 6 & 10 & 15 & 7 & 10 & 4 & 4 & 3 & 62 \\
\hline $\begin{array}{l}\text { Phytothérapeute } \\
\text { s }\end{array}$ & - & 2 & 3 & 4 & - & 1 & 2 & - & - & 12 \\
\hline Consommateurs & 8 & 4 & 6 & 8 & 3 & 4 & 6 & 5 & 2 & 46 \\
\hline $\begin{array}{l}\text { Nombres } \\
\text { d'enquêtés par } \\
\text { village }\end{array}$ & 15 & 17 & 27 & 36 & 16 & 20 & 20 & 12 & 7 & 170 \\
\hline Total & & & 95 & & & & 75 & & & \\
\hline
\end{tabular}

Source : Enquête de terrain, avril, 2016

Les données collectées sont relatives aux catégories d'usage des Produits Forestiers Non Ligneux, aux connaissances médicinales liées à l'utilisation de ces produits, à la disponibilité saisonnière des produits, aux interdits et cérémonies ou rites liés aux PFNL.

Parallèlement aux enquêtes, des prospections ont été exécutées dans l'ensemble de ces villages riverains avec un ou plusieurs guides locaux ayant de solides connaissances locales en pharmacopée traditionnelle afin de vérifier l'existence ou non des espèces végétales citées par les populations.

\subsection{Méthodes de traitement des données}

\subsubsection{Identification des espèces}

Toutes les espèces végétales présentes dans les placeaux installés au sein du massif forestier d'Agoua ont été récoltées et mises en herbier. Ces herbiers ont été ensuite identifiés à l’Herbier National du Bénin (Akoègninou 
et al. 2006). Les espèces une fois identifiées ont été réparties en diverses familles. Parmi les espèces collectées, celles servant de PFNL ont été recensées grâce aux enquêtes auprès des populations riveraines de la forêt.

\subsubsection{Calcul du taux de réponse et de la valeur d'usage ethnobotanique}

Les informations sur les formes d'utilisations des PFNL ont été regroupées par villages, en fonction du groupe socioculturel, du sexe et de la catégorie d'âge. Après le dépouillement et l'encodage des données dans une matrice Excel, différents taux, tests de relation ou de comparaison ont été calculés.

\section{- Taux de réponse}

Le taux de réponses par type d'utilisation est exprimé par la formule suivante :

\section{$F=100 S / N$}

Avec $\mathrm{F}$ : taux de réponse donné ;

$\mathrm{S}$ : nombre de personnes ayant fourni une réponse positive par rapport à une utilisation donnée et $\mathrm{N}$ : nombre de personnes interviewées.

- Valeur d'usage ethnobotanique

Le calcul de la valeur d'usage ethnobotanique des Produits Forestiers Non Ligneux est effectué à l'aide de la formule suivante, définie par Lykke (2004) :

$\boldsymbol{v} \boldsymbol{u}(\boldsymbol{i})=\frac{\sum_{i}^{n} s \bar{i}}{n} \quad$ Avec

$v u(i)$ : la valeur d'usage de l'espèce pour une catégorie donnée ;

si : le score d'utilisation attribué par les répondants

$\mathrm{n}$ : est le nombre de répondants pour une catégorie d'usage

L'intérêt de la valeur d'usage ici est qu'elle permet de déterminer de façon significative la catégorie d'usage ayant une grande valeur d'utilisation.

Le tableur Excel 2007 a été utilisé pour calculer les scores moyens et ensuite, ces valeurs sont regroupées par catégories pour tester l'existence ou non d'une différence significative entre leur appréciation selon les groupes socioculturels, les sexes, les villages et les différentes catégories d'âge des personnes interviewées. Pour ce faire, le test d'analyse de variance (ANOVA) est réalisé et lorsque les conditions d'application de ANOVA n’ont pas été vérifiées (normalité, homogénéité des variances), le test non paramétrique de Kruskal Wallis est effectué pour tester l'existence ou non d'une différence significative entre les catégories sus-citées. Ces différents traitements ont été effectués dans le logiciel MINITAB version 14. 


\section{2- Résultats}

\subsection{Inventaire des Produits Forestiers Non Ligneux exploités par les populations}

Les investigations de terrain ont permis d'inventorier 105 espèces végétales exploitées par les groupes socioculturels des massifs d'Agoua. Ces espèces sont réparties en 48 familles, soit une moyenne d'environ 2,187 espèces par famille. La figure 3 présente les espèces utilisées comme PFNL par les populations riveraines du massif d'Agoua selon les familles les plus représentatives.

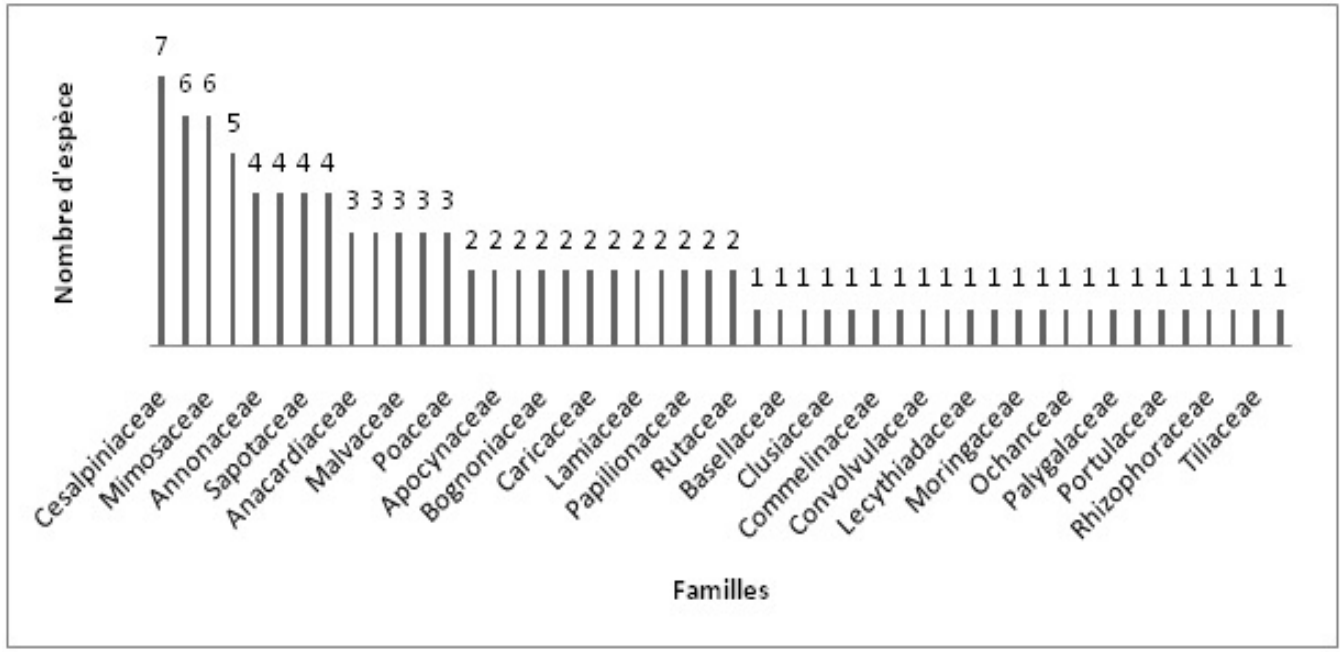

Figure 3 : Espèces utilisées comme PFNL par les populations riveraines du massif d'Agoua Source : Résultats des travaux de terrain, avril, 2016

L'analyse de la figure 3 montre que les familles les plus sollicitées sont les Césalpiniaceaes avec 7 espèces (6,66 \% du total), les Fabaceae et Mimosaceaes (6 espèces, 5,71\%), les Asteraceaes $\quad$ (5 espèces, 4,76\%), les Annonaceaes, les Euphorbiaceaes, les Sapotaceae, les Verbenaceae (4 espèces, 3,81\% chacune), les Anacardiaceae, les Gramineae, les Malvaceae, les Meliaceae et les Poaceaes (3 espèces, 2,85\% chacune). Par contre, les familles les moins sollicitées sont les Basellaceae, Moringaceae, Pontédériaceae, Portulaceae, Tiliaceae et Vitaceae.

\subsection{Identification des zones de prélèvement des espèces et types bio morphologiques}

La figure 4 présente les résultats de l'identification des zones de prélèvement des espèces utilisées comme PFNL dans le massif forestier d'Agoua. 


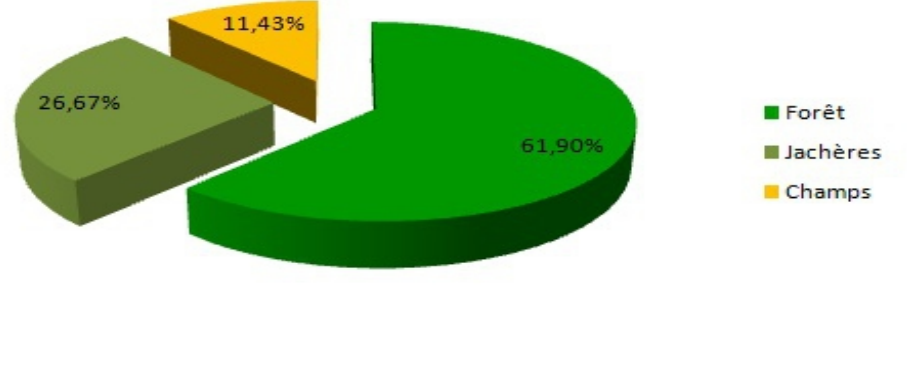

Figure 4 : Zones de prélèvement des espèces par les populations dans le massif d'Agoua

Source : Résultats des travaux, Avril, 2016

Il ressort de l'analyse de la figure 4, que pour l'ensemble des groupes socioculturels du massif forestier d'Agoua, c'est dans la forêt que les espèces servant de PFNL sont le plus récoltées (61,90 \% soit 65 espèces). Les jachères constituent la deuxième zone de prélèvement $(26,67 \%$ soit 28 espèces) et les champs représentent la zone de prélèvement de 11,43 \% (12 espèces). Suivant les types biomorphologiques, les espèces herbeuses sont les plus récoltées par les populations riveraines (70 espèces soit 66,67 \%). Les arbres représentent 19,04 \% (20 espèces) des espèces récoltées et les arbustes quant à eux représentent 14,28 \% (15 espèces) des PFNL récoltées.

\subsection{Différentes catégories d'utilisation des PFNL}

Les résultats des enquêtes de terrain ont montré que les riverains des massifs d'Agoua utilisent les différentes espèces végétales qu'ils récoltent dans six (6) domaines à savoir la médecine traditionnelle, l'alimentation, l'usage domestique, les matériaux de construction, l'emballage et les colorants (Tableau 2).

Tableau 2 : Catégories d'usages des PFNL

\begin{tabular}{cr}
\hline Catégories d'utilisations & Pourcentage(\%) \\
\hline Médecine traditionnelle & 58,09 \\
Alimentation & 28,57 \\
Usage domestique & 26,66 \\
Matériaux de constructions & 7,61 \\
Emballage & 5,71 \\
Colorants & 3,8 \\
\hline
\end{tabular}

Source : Résultats des travaux, Avril, 2016

Il ressort du tableau 2 que 61 espèces sont utilisées dans la médecine traditionnelle (58,09\%), 30 espèces servent dans l'alimentation (28,57 \%), 
28 espèces sont utilisées pour l'usage domestique (26,57 \%), 8 espèces comme matériaux de construction (7,61 \%), 6 espèces servent comme emballage $(5,71 \%)$ et 4 espèces sont utilisées comme colorant $(3,8 \%)$. Le test de variance ANOVA effectué sur les données montre qu'il y a une différence significative $\left(\mathrm{R}^{2}=45,54 \%, \mathrm{P}=0,01\right)$ entre les espèces et les catégories d'utilisation. Les organes utilisés varient également d'une espèce à une autre. En effet, ce sont les feuilles, les fruits, les écorces, les fleurs, et les racines qui sont utilisées par les populations riveraines du massif forestier d'Agoua.

\subsubsection{Usages des espèces végétales dans l'alimentation et la médecine traditionnelle}

Les PFNL entrent dans l'alimentation des populations riveraines du massif forestier d'Agoua. En effet, 30 espèces ont été identifiées comme très utiles pour la satisfaction de leurs besoins alimentaires. Sur ces 30 espèces, 12 sont utilisées par les populations sous forme de fruits (40\%), 4 espèces $(13,33 \%)$ sont consommées sous forme de sauce légume gluante et 6 (20 \%) sont consommés sous forme de sauce légume non gluante. Certaines espèces telles que Sorghum vulgare, Ocimum basilicum sont également employées comme additifs sous forme d'épices d'assaisonnement. Ces divers Produis Forestiers Non Ligneux aident à assurer les besoins alimentaires des populations riveraines du massif forestier d'Agoua voire de toute la commune de Bantè.

En dehors de l'usage alimentaire des espèces végétales, les populations locales les utilisent également dans la médecine traditionnelle

Ainsi, les maladies courantes telles que le paludisme, la diarrhée, les maux de ventre, les maux de tête, le vomissement et la fièvre sont les plus traitées au sein des ménages, grâce aux PFNL. L'absorption de ces produits s'effectue soit par décoction, par infusion ou par une autre médication. Les parties des plantes utilisées pour le traitement des maladies courantes sont les fruits, les écorces, les racines et les feuilles.

Les populations peuvent avoir recours à plusieurs espèces pour traiter une même maladie. Une même espèce peut également être utilisée pour traiter différentes maladies. La vertu des plantes constitue un secret dont seuls les guérisseurs traditionnels et certains chefs de religion traditionnelle détiennent la connaissance. Ces derniers ne révèlent le secret des plantes qu'à leurs fils, leurs fidèles, à des sages ou à des initiés. Mais à partir des révélations de certaines personnes enquêtées, les vertus thérapeutiques de certaines espèces ont été obtenues. Ainsi, il est noté que 23 espèces sont utilisées dans le traitement du paludisme, 12 pour le traitement des maux de ventre, 11 pour le bain du nouveau-né, 6 dans le traitement de la teigne, 4 
dans le traitement de la toux, 3 espèces pour le traitement de la tension artérielle, etc.

Les formes sous lesquelles les espèces végétales sont utilisées en pharmacopée varient suivant la maladie. Les formes courantes sont la décoction et l'infusion pour les racines et les écorces dans le cas du traitement des mammites. Pour les fruits de Adansonia digitata utilisés pour susciter la lactation chez la nourrisse, c'est la poudre qui est surtout recherchée. Le beurre de karité qui est obtenu des noix de Vitellaria paradoxa sert souvent de liant dans l'application des poudres obtenues de racines et écorces après séchage. Pour une meilleure efficacité de ces différents produits, ils sont parfois utilisés en association.

\subsubsection{Autres utilisations des espèces végétales}

Le bois de chauffe est la matière principale utilisée par les populations comme source d'énergie. A cet effet, les espèces comme Cola millenii, Dialium guineense, Psidium guajava, Albizia glaenima, Cassia Alata, etc. sont les plus utilisées.

Une faible proportion (8\%) des espèces recensées est également utilisée dans l'artisanat (Piliostigma thonningui ; Andropogon schirensis ; Dioscorea abyssinica ; Raphia sudanica ; Bombax costatum...), pour les cordes et la brosse végétale. Les plantes tinctoriales sont également exploitées, mais à des fins d'autoconsommation ou de petit commerce. Outre ces utilisations, certaines espèces recensées, environ 5\%, servent comme charpente dans la construction des bâtiments (Afzelia africana, Khaya senegalensis, Céiba pentanda, Pterocarpus erinaceus, Anacadius occidentale).

\subsubsection{Mode de récolte des PFNL}

Le mode de récolte des espèces végétales pourvoyeuses des PFNL, est fonction des organes à utiliser. Ces organes varient également d'une espèce à une autre. Ce mode de récolte peut affecter l'abondance de l'espèce dans le milieu naturel puisqu'il existe une relation manifeste entre la partie utilisée de la plante et les effets de cette exploitation sur son existence (Cunningham, 1996). En effet, les 105 espèces d'utilité socioculturelle identifiées dans le massif d’Agoua sont recherchées soit pour leurs feuilles, leurs racines, leurs écorces, leurs fruits, leurs pépins. La figure 5 montre les proportions d'espèces utilisées par organe. 


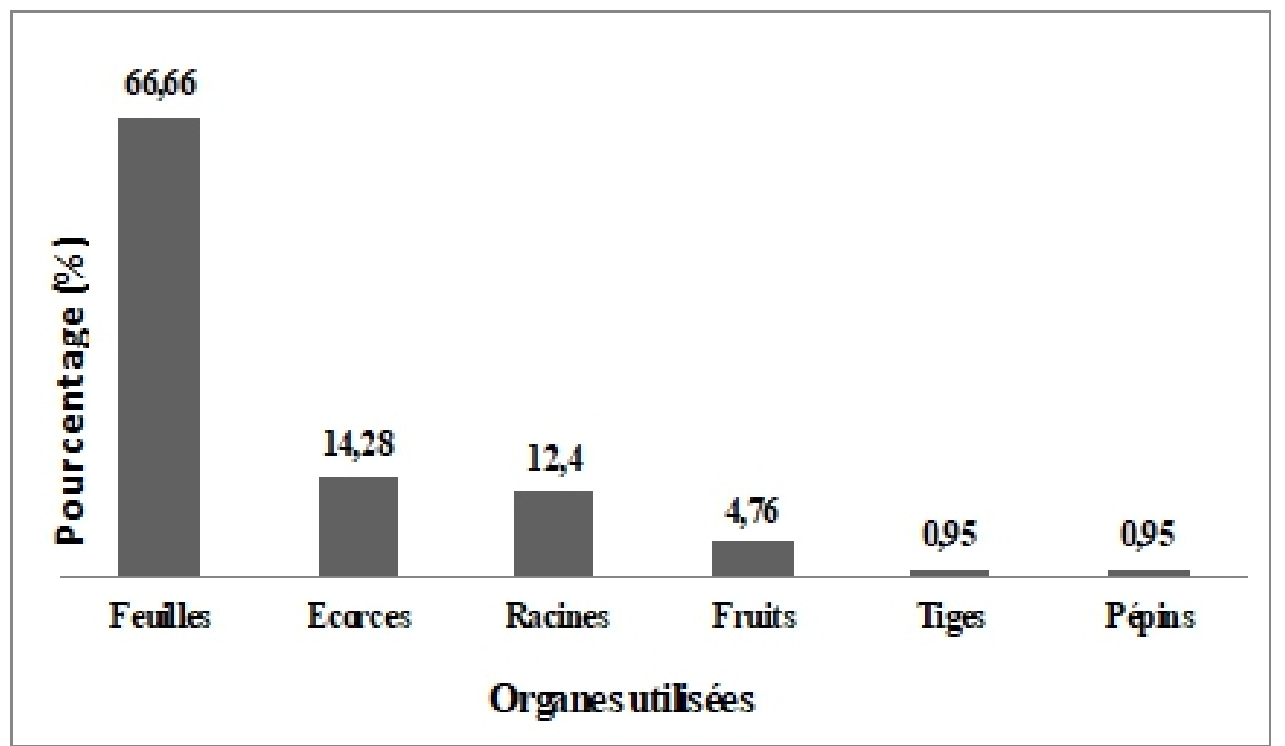

Figure 5 : Proportion des PFNL utilisés par organe

Source : Résultats des travaux, Avril 2016

Les espèces pourvoyeuses des PFNL dans le massif forestier d'Agoua sont diversifiées. L'analyse de la figure 5 montre que 66,66 \% de ces espèces sont exploitées pour leurs feuilles ; 14,28 \% d'espèces ligneuses sont exploitées pour leurs écorces et $12,40 \%$ pour leurs racines. Ces organes sont les plus utilisés, sur les espèces végétales collectées, par les populations riveraines du massif forestier d'Agoua. Le mode de récolte varie d'un simple prélèvement à l'arrachage de la plante entière en fonction de l'organe recherché et de la taille de l'individu ou de l'espèce. Ce potentiel varié des organes prélevés sur les espèces pourvoyeuses constitue une source de bienêtre et permet une diversification dans l'alimentation dans la Commune de Bantè en général et dans le milieu d'étude en particulier.

\section{3- Discussion}

Au Bénin en général et dans les régions du massif forestier d'Agoua en particulier, du fait de leurs usages multiples dans les domaines de l'alimentation, de la construction, de l'énergie domestique et de la technologie locale, de la médecine traditionnelle et du mysticisme (Bergeret et Ribot, 1990 ; Biaou, 1996 ; Assogbadjo 2000), les espèces végétales pourvoyeuses de PFNL occupent une place très importante dans la vie socioculturelle des populations locales. Ce constat a été effectué par plusieurs chercheurs aussi bien nationaux qu'internationaux qui se sont intéressés aux PFNL sous divers angles. Ainsi, les travaux de Bonou (2008), ont montré que les ressources naturelles contribuent de façon significative à 
la subsistance en milieu rural et à l'économie nationale. Lawani (2007) abonde dans le même sens en démontrant l'importante contribution du bois énergie aux moyens d'existence durable des ménages riverains de la réserve de biosphère de la Pendjari. Par ailleurs, Diop et al (2005) à partir d'une étude ethnobotanique, essentiellement qualitative, ont conclu que la connaissance des préférences des populations rurales en ressources végétales forestières est une donnée importante pour la définition des politiques de gestion et de conservation des ressources naturelles et pour la mise en œuvre des plans d'aménagement forestier. Des enquêtes ethnobotaniques ont également permis à Lougbégnon et al (2011), de connaître l'importance d'utilisation et la pression qui s'exerce sur les espèces végétales de la vallée du Sitatunga. Les résultats de leurs travaux ont montré que les populations riveraines de la réserve sont conscientes des multiples avantages que les 74 plantes utiles identifiées dans la réserve leur offrent et perçoivent les risques relatifs à leur dégradation. Cependant, elles continuent de faire pression sur ces espèces importantes sur le plan des usages, qui tendent à disparaître écologiquement de la forêt. En effet, l'action prédatrice de l'homme s'est imposée comme une nouvelle composante de la dynamique progressive et/ou régressive de la végétation (Klassou, 1996). De plus, en l'absence d'une réglementation de la récolte des PFNL en forêt, une forte pression de récolte peut donc conduire à la réduction et/ou la perte de la biodiversité, la diminution de la productivité, etc. Il est donc nécessaire de mettre en place une stratégie s'inscrivant dans l'optique d'une exploitation durable des ressources forestières et bien d'autres services (Guédjé et al, 2003 ; Bonou, 2008).

\section{Conclusion}

La présente étude a permis d'inventorier les Produits Forestiers Non Ligneux (PFNL) du massif forestier d'Agoua et de déterminer leurs valeurs d'usage pour les populations riveraines. En raison des conditions biophysiques favorables, le massif forestier d'Agoua, outre la ressource bois d'œuvre, possède de grandes potentialités en matière de PFNL. L'exploitation de ces espèces végétales pourvoyeuses des Produits Forestiers Non Ligneux contribue au bien-être des populations riveraines et de celles des autres localités de la Commune de Bantè. Environ $80 \%$ de la population utilisent ces ressources au quotidien pour satisfaire leurs besoins de subsistance. Que ce soit au niveau local, régional, national et international, les PFNL procurent de la nourriture, des plantes médicinales, des plantes ornementales, de l'énergie, des matériaux de construction, des biens et des ustensiles divers aux populations. Ils ont aussi une grande valeur socioculturelle et religieuse. Vu l'important rôle que jouent les PFNL dans la 
vie quotidienne des populations locales, des stratégies doivent être définies pour leur gestion et leur exploitation durables.

\section{References:}

1. Adam S., Boko M. (1993) : Le Bénin. Les éditions du Flamboyant/EDICEF, 96 p.

2. Akoègninou A., Van der Burg W. J., Van der Maesen L. J. G. (2006): Flore Analytique du Bénin. Leinden, Backhuys, 1034 p.

3. Arouna O. (2012) : Cartographie et modélisation prédictive des changements spatio-temporels de la végétation dans la Commune de Djidja. Thèse de Doctorat Unique, Université deeAbomey-Calavi. Ecole Doctorale Pluridisciplinaire « Espace, Cultures et Développement », 246 p.

4. ASECNA (2016) : Station météorologique de Bantè, Agence pour la Sécurité de la Navigation Aérienne en Afrique et à Madagascar.

5. Assogbadjo A. E. (2000) : Etude de la biodiversité des ressources forestières alimentaires et évaluation de leur contribution à l'alimentation des populations locales de la forêt classée de la Lama. Thèse d'ingénieur agronome, FSA)-UNB, 131p.

6. Bergeret A., Ribot J. C., (1990) : L’arbre nourricier en pays sahélien. Paris, France, Éditions de la Maison des sciences de l'homme, 237 p.

7. Biaou G. (1996) : Pouvoir local et gestion des ressources naturelles. Cotonou, Bénin, 8p.

8. Bonou A. (2008) : Estimation de la valeur économique des Produits Forestiers Non Ligneux (PFNL) d'origine végétale dans le village de Sampéto (commune de Banikoara). Mémoire du D.E.A, Abomey Calavi, FSA-UAC, 66 p.

9. Braun Blanquet. (1932): Plant sociology- The study of plant commuties- translatedrevised and edited by Fuller G.D. Conard H. S. $439 \mathrm{p}$.

10. CeCPA Bantè (2015) : Rapport et plan de campagne agricole dans la commune de Bantè, commune de Bantè, République du Bénin, 34 p.

11. Colyn M., Oudé A. P., Natta A. K., Fonton Kiki B. (2007) : Etude chasse villageoise de subsistance (ECVS) dans la zone d'intervention du projet d'aménagement des forêts classées des Monts Kouffé, de Wari-Maro et d’Agoua au centre Bénin: Etude pilote du PAMF (DGFRN/MEPN) de Mai 2006 à Juin 2007, Cotonou, Bénin, 143 p.

12. Cunningham. (1996): La gestion participative des ressources naturelles dans le bassin du Congo: l'exemple de la République Démocratique du Congo. Mémoire de maitrise, Université de Limoges, 86 p.

13. de Souza S. (1988): Flore du Bénin. Noms des plantes dans les 
langues nationales béninoises. Tome 3, 424 p.

14. Diop M., Kaya B., Niang A., Olivier A. (2005) : Les espèces ligneuses et leurs usages : les préférences des paysans dans le Cercle de Ségou, au Mali. Nairobi, Kenya, World Agroforestry Centre (Icraf), Working Paper n 9, 26 p.

15. Djego J. G. M. (2006) : Phytosociologie de la végétation de sous-bois et impact écologique des plantations forestières sur la diversité floristique au Sud et au Centre du Bénin. Thèse de

16. Doctorat, Université d’Abomey-Calavi, 388 p.

17. Fandohan B, Assogbadjo A. E., Glèlè Kakaï R., Kyndt T., De Caluwé E., Codjia J. T. C., Sinsin B. (2010): Women's Traditional Knowledge, Use Value, and the Contribution of Tamarind (Tamarindus indica L.) to Rural House holds’ Cash Income in Benin. Economic Botany, 64: 248-259.

18. Guédjé P., Delis C. (2003): L’évaluation des impacts sur l'environnement: processus, acteurs et pratique pour un développement durable. $3^{\mathrm{e}}$ Edition, $240 \mathrm{p}$.

19. INSAE (2013): Résultats provisoires du RGPH 4, Ministère du Développement, de l'Analyse Economique et de la Prospective, République du Bénin, 8 p.

20. INSAE (2003) : Troisième Recensement Général de la Population et de l'Habitation (RGPH 3). Février 2002. Synthèse des analyses en bref, Cotonou, Bénin, 48 p.

21. Klassou K. S. (1996) : Evolution climato-hydrologique récente et conséquence sur l'environnement : l'exemple du bassin versant du fleuve mono (TOGO-BENIN). Thèse de doctorat de l'université Michel de Montaigne-Bordeaux III : $471 \mathrm{p}$.

22. Lawani A. (2007): Contribution du bois énergie aux moyens d'existence durables des ménages riverains de la Réserve de Biosphère de la Pendjari. Mémoire, Université d’Abomey-Calavi (UAC), Bénin, 133 p.

23. Lokonon C. B. (2002) : Valeur économique des Produits Forestiers Non Ligneux (PFNL) au Bénin. Vertigo- la revue électronique en sciences de l'environnement (Online), Volume 07 Numéro 1/ avril 2002, mise en ligne consulté le 16 avril 2002.Consulté le 25 mai 2015.URL : http :// vertigo.revues.org/2813;DOI : 10.400/vertigo.13500.

24. Lougbegnon T. O., Tente B. A. H., Amontcha M., Codjia J. T. C. (2011): Importance culturelle et valeur d'usage des ressources végétales de la réserve forestière marécageuse de la vallée de Sitatunga et zones connexes. Bulletin de la Recherche Agronomique du Bénin Numéro 70-Décembre 2011. 
25. Lykke A. M., Kristensen M. K., Ganaba S. (2004): Valuation of the local dynamics of 56 woody species in the Sahel. Biodiversity and Conservation 13: pp.1961-1990.

26. Oumorou M. (2003): Etudes écologique, floristique, phytogéographique et phytosociologique des inselbergs du Bénin. Thèse de doctorat, Université Libre de Bruxelles, 210 p + annexe.

27. PAMF (2007) : Plan d'aménagement participatif de la forêt classée d’Agoua. Cotonou, Bénin, 244p.

28. Riley B. W., Brokensha D. (1988): The Mbeere in Kenya. 2vol. New York, University of America Press.

29. Sinsin B. (1993) : Phytosociologie, écologie, valeur pastorale et capacité de charge des pâturages naturels du périmètre Nikki-Kalalé au Nord-Bénin. Thèse de Doctorat en sciences agronomiques, Section interfacultaire d’Agronomie et de Phytosociologie, Université Libre de

30. Belgique, $390 \mathrm{p}$.

31. Sokpon N., Lejoly J. (1996) : Les plantes à fruits comestibles d'une forêt semi-caducifoliée de Pobè au sud-est du Bénin. L’alimentation en forêt tropicale: interactions bioculturelles et perspectives de développement. Annales de la Faculté des Sciences de Kisangani (1) : 115-124.

32. Toko I. (2008): Etude de la variabilité spatiale de la biomasse herbacée, de la phénologie et de la structure de la végétation le long des toposéquences du bassin supérieur du fleuve Ouémé au Bénin. Thèse de Doctorat, Université d’Abomey-Calavi, Bénin, 241 p.

33. Wotto D. (2013) : Importance socio-économique et culturelle des produits forestiers non ligneux chez les populations riveraines des massifs forestiers d'Agoua. Mémoire de DEA, UAC/EDP/FLASH, $82 \mathrm{p}$.

34. Yelkouni M. (2004): Gestion d'une ressource naturelle et action collective : le cas de la forêt de Tiogo au Burkina Faso. Thèse de doctorat en sciences économiques, 248 p. 